\title{
A Theoretical and Experimental Study of Viscoelastic Rolling Contacts Incorporating Thermal Effects
}

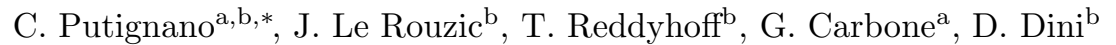 \\ ${ }^{a}$ TriboLAB-Dipartimento di Ingegneria Meccanica e Gestionale, Politecnico di Bari, V.le \\ Japigia 182, 70126 Bari-ITALY. \\ ${ }^{b}$ Department of Mechanical Engineering, Imperial College London, Exhibition Road, South \\ Kensington, London,SW7 2AZ, United Kingdom.
}

\begin{abstract}
Viscoelastic contacts are present in countless industrial components including tires, dampers and rubber seals. The effective design of such components requires a full knowledge of viscoelastic contact mechanics in terms of stresses, strains and hysteric dissipation. To assess some of these issues, this paper describes a series of experiments on the contact area and penetration in a rolling contact between a nitrile rubber ball and a glass disk. The experimental results are compared with the theory proposed by Carbone and Putignano in [1] showing close agreement at low speeds. However, discrepancies arise at speeds above $100 \mathrm{~mm} / \mathrm{s}$ because of the frictional heating. In order to evaluate this effect, the temperature of the sliding interface is measured for different rolling speeds using infrared microscopy. Thermal results showed that interfacial temperature remained constant at low rolling speeds before rising significantly when speeds above $100 \mathrm{~mm} / \mathrm{s}$ were reached. These temperature effects are incorporated into the numerical simulations by means of an approximated approach, which corrects the viscoelastic modulus based on the mean measured temperature in the contact. The result of this approach is to extend the region of agreement between experimental and numerical agreement to higher speeds.
\end{abstract}

Keywords: viscoelastic contact mechanics, contact heating, contact mechanics measures.

\section{Introduction}

Because of their increasingly widespread use in many industries, polymers have considerable prominence in modern engineering research. Currenly, much attention is paid to soft matter mechanics and, in particular, to improve our understanding of problems involving surface interactions. Soft material mechanics is, in fact, crucial for a number of engineering applications including tyres and

\footnotetext{
*Corresponding author. Phone: +39 080596 2701; fax: +39 0805962777

Email address: c.putignano@poliba.it (C. Putignano)
} 
seals ([2] [3]) , bioengineering [4], nanotechnology [5] and "green" technology [6]. The behaviour of these components is complicated by viscoelastic contact mechanics. This point is especially important in applications involving rolling and sliding contacts. In such cases, because of the hysteretic deformation of soft components, there is a viscoelastic dissipation (defined here as viscoelastic friction) in addition to interfacial friction. This increases the mechanical energy loss $([7],[8],[9],[10],[13],[14],[15])$, which has to be correctly accounted for so that the energy savings required by modern design can be achieved. Furthermore, viscoelasticity entails a strong dependence of contact area, penetration and, therefore, contact stiffness on the speed of motion. This, for example, may have a paramount importance in seals where fluid percolation is closely related to the real contact area([2] [3]). Finally, due to the marked dependence of the viscoelastic properties on the material temperature, the thermal heating caused by sliding or rolling motion should be quantified in order to to optimize the components design [12].

Because of the relevance of these themes, many scientific investigations have been carried out from analytical, numerical and experimental points of view ([7], [8], [9],[10],[13],[14], [15]). Pioneering analytical approaches to the problem were proposed by Hunter [7] and, independently, by Goriacheva [16] for the case of two-dimensional (2D) contacts, i.e. for a rigid cylinder in contact with a viscoelastic half-space. An alternative analytical methodology was developed by Lee and Radok [17], who extended the viscoelastic correspondence principle to solve the Hertzian viscoelastic contact problem. One of the main limitations, affecting these analytical theories and the following developments, is related to the particular shape of the contact punch for which the problem is solved. Furthermore, these models are constrained to the simplest form of viscoelasticity, i.e. to materials with only a single relaxation time. Unfortunately, as shown in $([1],[18])$, real viscoelastic materials exhibit complex mechanical behavior, marked by a large spectrum of relaxations times. Consequently, these techniques are not able to produce quantitative results for real cases. In the last decade, Persson ([10], [11]) proposed new approaches capable of being applied - at least in approximate forms - to a general viscoelastic material. Furthermore, he has also investigated the role of thermal heating in the sliding motion between rough surfaces [12]. However, to the authors' knowledge, no definitive study specifically related to thermal effects in rolling viscoelastic contact has been produced.

Owing to these limitations, a variety of numerical approaches has been developed. Boundary Element Methods (BEM), as in [15], can provide accurate results, but on the other hand, these methodologies are usually only able to manage ideal viscoelastic materials with a single relaxation time. On the other hand, Finite Element Methods (FEM) ([14],[19] ,[20], [21], [22]) can handle real viscoelastic materials, but, since they have been conceived mainly for structural modeling, accuracy in the contact solution is insufficient. This problem assumes heavy proportions in the case of sliding or rolling contact between rough surfaces. Furthermore, the very large number of length and time scales involved in the problem makes FE models unsuitable due to the current computational 
resources [1].

A number of experimental methodologies have been developed to measure the main parameters marking soft contact mechanics. After the pioneering attempt proposed by Grosch [9], numerous techniques have been proposed to measure the viscoelastic friction([1], [8], [26], [32], [33],[30]). Several attempts have also been developed to catch the contact area ([8], [24], [25], [26], [30]). However, these are typically focused on elucidating other phenomena and have usually been developed under low speeds and loads conditions. Specifically, these contributions analyze the role of the adhesion force ([25], ,27]) aiming to take into account the surface roughness; however, viscoelastic effects, such large changes in the shape and size of the contacting area, have not been quantitatively investigated. Furthermore, apart from the pioneering attempt by Wannop and Archard who tried to measure the sub-surface temperature by means of thermocouples [28], to the best of the authors' knowledge, no modern thermal analysis of the contact area has been presented so far. Consequently, there is little understanding of the importance of the frictional heating in viscoelastic rolling contact.

In this paper, we present some techniques that may address these unsolved issues. In particular, in the case of a rolling contact between a rubber ball and a glass disk, we measure the contact area and penetration dependence on the speed of motion. This enables us to compare experimental data with numerical predictions of the theory presented in [1]. Furthermore, a thermal analysis allows us to obtain the temperature distribution in the contact area and let us understand at which speeds thermal heating can be neglected without significant errors. The paper is set out as follows. Section 2 outlines the proposed viscoelastic model and the mathematical formulation developed to carry out the numerical predictions. Section 3 then contains the experimental procedures employed to measure penetration, contact area and temperature. Finally, in Section 4, the experimental outcomes are shown, discussed and compared with numerical simulations. The last Section includes conclusions and closing remarks.

\section{Numerical Formulation}

The viscoelastic response of linear rubber-like materials can be described by the following integral equation ([18] [29]):

$$
\varepsilon(t)=\int_{-\infty}^{t} d \tau \mathcal{J}(t-\tau) \dot{\sigma}(\tau),
$$

where $\varepsilon(t)$ is the time-dependent strain, $\sigma(t)$ is the stress (the symbol '.' stands for the time derivative), and the function $\mathcal{J}(t)$ is the creep function that must satisfy causality, i.e. $\mathcal{J}(t<0)=0$. The most general form of $\mathcal{J}(t)$ is given by [18]:

$$
\mathcal{J}(t)=\mathcal{H}(t)\left[\frac{1}{E_{0}}-\int_{0}^{+\infty} d \tau \mathcal{C}(\tau) \exp (-t / \tau)\right],
$$


where $\mathcal{H}(t)$ is the Heaviside step function, the real quantity $E_{0}$ is the rubber elastic modulus of the material at zero-frequency, $\mathcal{C}(\tau)$ is a strictly positive function usually defined as the creep (or retardation) spectrum( [18] [29]), and $\tau$ is the relaxation time, continuously distributed on the real axis.

In the numerical procedure we are developing, Eq. (2) has to be discretized by assuming $\mathcal{C}(\tau)=\sum_{k} C_{k} \delta\left(\tau-\tau_{k}\right)$ and, therefore, rewritten as:

$$
\mathcal{J}(t)=\mathcal{H}(t)\left[\frac{1}{E_{0}}-\sum_{k=1}^{n} C_{k} \exp \left(-t / \tau_{k}\right)\right] .
$$

As commonly suggested in the literature [18] [29], we take the Fourier transform of the equations presented above. In particular, since Eq. (2) can be Fourier transformed as $\varepsilon(\omega)=\sigma(\omega) / E(\omega)$ with $E(\omega)=[i \omega J(\omega)]^{-1}$, we can easily write the following equation for the viscoelastic transformed complex modulus $E(\omega)$ :

$$
\frac{1}{E(\omega)}=\frac{1}{E_{0}}-\int_{0}^{\infty} d \tau \frac{i \omega \tau \mathcal{C}(\tau)}{1+i \omega \tau}=\frac{1}{E_{\infty}}+\int_{0}^{\infty} d \tau \frac{\mathcal{C}(\tau)}{1+i \omega \tau}
$$

Such an expression for $E(\omega)$ has a prominent physical meaning. As shown in Fig. 1, at 'low' frequencies the material is in the 'rubbery' region. Here, $E_{1}=\operatorname{Re} E(\omega)$ is relatively small and approximately constant, while the imaginary part $E_{2}=\operatorname{Im} E(\omega)$ is also small, although it increases very rapidly: consequently, the viscoelastic dissipation becomes negligible. On the other hand, at very high frequencies the material is elastically very stiff (brittle-like). In this 'glassy' region $E_{1}(\omega)$ is again nearly constant but clearly much larger than in the rubbery region. Finally, in the intermediate frequency range (the so called 'transition' region), the loss tangent $\operatorname{Im} E(\omega) / \operatorname{Re} E(\omega)$ is very large [see Fig. (b)] and consequently we find the energy dissipation during sliding and/or rolling motion.

Finally, it should be noted that temperature has a dramatic effect on the viscoelastic properties. For the majority of linear viscoelastic materials, including many rubbers and many rubber-based composites, it is possible to assume a very simple logarithmic relation between temperature and time. Indeed, the effect of changing the temperature is simply to shift the viscoelastic frequency response (plotted against log frequency) along to the frequency axis. This then entails shifting to the right the viscoelastic modulus since, due to the thermal energy, the material tends to relax. Therefore, a time-temperature shift factor $a_{T}(T)$ can be defined to calculate, given the $n$ relaxation times $\tau_{k}$ at a reference temperature $T_{r e f}$, the new values for these parameters at a given temperature T:

$$
\log \left(a_{T}\right)=\log \tau_{k}(T)-\log \tau_{k}\left(T_{\text {ref }}\right) \quad k=1, \ldots ., n
$$

A empirical expression for $a_{T}$ is suggested by Williams, R.F. Landel, and J.D. Ferry in [41] and is valid for many real viscoelastic materials. 


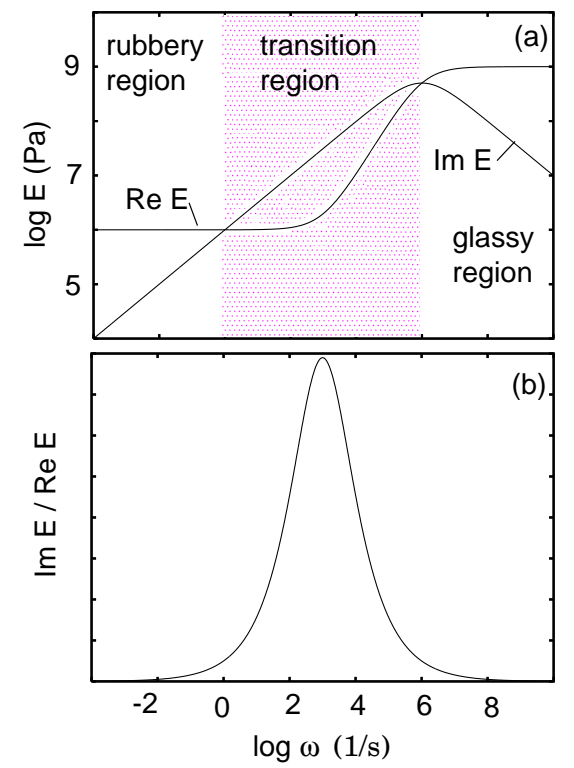

Figure 1: The real $E_{1}=\operatorname{Re}[E(\omega)]$ and the imaginary $E_{2}=\operatorname{Im}[E(\omega)]$ parts of the viscoelastic modulus $E(\omega)$ of a typical rubber-like material, (a); The loss tangent $E_{2}(\omega) / E_{1}(\omega)$, (b).

Now, by moving from the Green function approach already developed in ([46] [35] [36] [37]) and relying on the previous model for the viscoelastic materials, a new boundary element approach has been proposed to assess the mechanics of sliding and rolling viscoelastic contacts [1] . Basically, by recalling the translational invariance and the elastic-viscoelastic correspondence principle [18], we may formulate the general linear-viscoelastic contact problem between a rigid indenter and a viscoelastic slab as:.

$$
u(\mathbf{x}, t)=\int_{-\infty}^{t} d \tau \int d^{2} x \mathcal{J}(t-\tau) \mathcal{G}\left(\mathbf{x}-\mathbf{x}^{\prime}\right) \dot{\sigma}\left(\mathbf{x}^{\prime}, \tau\right),
$$

where $\mathbf{x}$ is the in-plane position vector, $t$ is the time, $u(\mathbf{x}, t)$ is the normal surface displacement of the viscoelastic solid, $\sigma(\mathbf{x}, t)$ is the normal interfacial stress, $\mathcal{J}(t)$ is given in Eq. (2) and the quantity $\mathcal{G}(\mathbf{x})$ is the Green's function. Now, assuming steady state conditions, i.e. sliding/rolling motion at constant velocity $\mathbf{v}$, it is possible to considerably simplify the problem. Indeed Eq. 6 can be re-written in the form:

$$
u(\mathbf{x}, \mathbf{v})=\int d^{2} X^{\prime} \mathcal{G}\left(\mathbf{x}-\mathbf{x}^{\prime}, \mathbf{v}\right) \sigma\left(\mathbf{x}^{\prime}\right)
$$

where the kernel $G(\mathbf{x}, \mathbf{v})$ depends parametrically on the motion speed $\mathbf{v}$.

For further details regarding the mathematical formulation, we refer the reader to [1], where the kernel $G(\mathbf{x}, \mathbf{v})$ is explicitly given. Here, we underline 
that, once a time-independent formulation is introduced, the problem can be solved by employing the same approach already developed for the linear elastic rough contact problem studied in ([35], [36], [37]).

\section{Experimental methodologies}

\subsection{Penetration and contact area}

To measure the penetration and the contact area, we develop the following experimental procedure. We employ a conventional EHD ball and disc rig (PCS Instruments Ltd, Acton, UK), in which a $19.05 \mathrm{~mm}$ diameter nitrile rubber ball (provided by The Precision Plastic Ball Co Ltd), is loaded against a glass disc, as shown in Fig. 2. Since the ball specimen has no shaft and is located on a roller carriage, when applying the load, the disc rotation drives under pure rolling contact conditions. Furthermore, in order to enable the accurate measurement of the displacements, we have incorporated additional components to the conventional EHL rig setup. In particular, a T shaped stem is attached to the ball loading system supporting the ball roller carriage. In such a way, a laser displacement sensor (LK-G32 produced by Keyence Ltd) is enabled to measure the vertical displacement corresponding to the penetration of the ball specimen. Clearly, the loading system is assumed to be considerably stiffer than the rubber ball specimen. Thanks to the high resolution of the displacement sensor $(0.05$ $\mu \mathrm{m})$, it is possible to get the small variations in penetration occurring when changing the rolling speed.

$[a]$

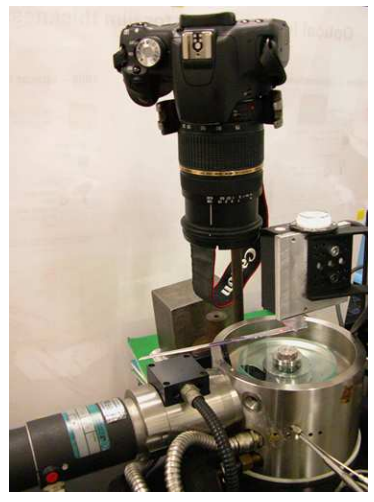

Camera

Laser Detector

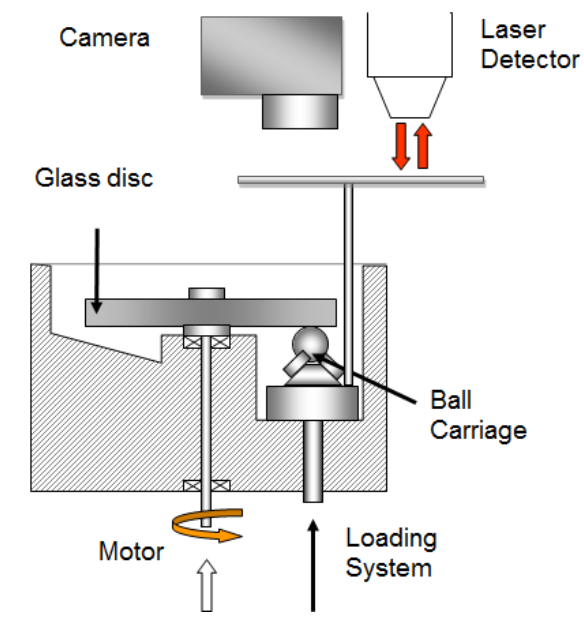

Figure 2: EHL test rig: (a) photograph of the experimental configuration; (b) schematic of the original setup and additional features used to accurately measure surface displacements.

Contact area measurements are obtained from images taken with a camera located above the glass disc (see Fig. 2). Due to the very low modulus of 
the rubber ball, contact area diameters are likely to be sizeable - i.e. several millimeters large - even under low loads. To capture such areas, an SLR camera (a Canon EOS 500D) and lens (a 7 Tamron AF 28-75mm f/2.8 XR Di LD) are employed.

Once the images are collected, an ad hoc MATLAB algorithm digitizes the contact area and extract its dimensions as they evolved owing to viscoelastic effects. After importing the black and white snapshots, each picture, managed as a matrix in the MATLAB environment, is Fourier-transformed and undergoes a filtering process, aimed at detecting the contact borders. The transition zone (i.e. the border between contacting and non-contacting regions) could then be found. Finally, the contact area was calculated knowing pixel size of the images (the latter being obtained from an image of a specimen of known dimensions, positioned at the contact interface).

\subsection{Thermal measurements}

The temperature distribution in the contact was measured using an infrared microscopy technique, developed in previous research [38],[39],[40]. This method uses the same apparatus described above to produce the contact (i.e. a nitrile ball, loaded against a transparent disc using a PCS interferometry rig); the only difference is that the glass disc had been replaced with a sapphire one. Since sapphire is transparent to infrared, this setup allowes the contact to be viewed from above using an infrared camera and microscope lens. The camera (a FLIR $\mathrm{X} 6540 \mathrm{SC}$ ) has a $640 \times 480$ focal plane array, which, in combination with the $3 \times$ infrared microscope lens, gives a resolution of $\approx 5 \mu \mathrm{m} /$ pixel. Calibration of the equipment involved correlating the counts detected by the camera to the temperature in the contact. This is achieved by gradually heating the ball, using cartridge heaters, and measuring the temperature using RTD probes (both located with the PCS EHL rig). Unlike previous research ([39],[40]), this approach , does not account for the background radiation from the bulk of the sapphire disc. However, it was assumed to be negligible, since the emissivity of sapphire is very low, especially when compared to the high emissivity of the nitrile ball specimen. Furthermore, these experiments are carried out unlubricated, so that heating of the sapphire disc is minimized due to there being no oil present to aid conduction.

\section{Results and discussion}

\subsection{Dynamic Mechanical Analysis}

The viscoelastic frequency response of the nitrile ball specimens is characterized by means of a Q800 Dynamic Mechanical Analyzer (DMA) manufactured by TA instruments. This equipment provides data over a set range of frequencies and temperatures. In details, fixed a pre-load equal to $7.5 \mathrm{~N}$, the test is carried out on rubber strips with cross section $2 \times 5.5 \mathrm{~mm}$ and lengh $30 \mathrm{~mm}$. The range of frequency is from 0.01 to $25 \mathrm{~Hz}$ and temperature varies from 0 to 100 degrees Celsius with $10{ }^{\circ} \mathrm{C}$ step. In order to extend the data to the very low frequencies 


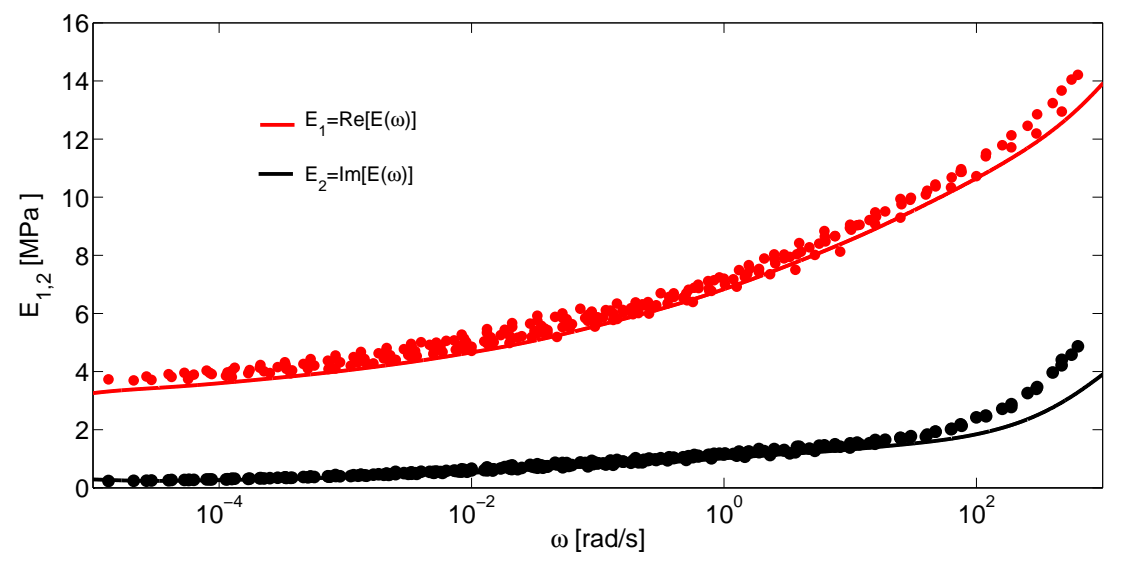

Figure 3: The real part $E_{1}=\operatorname{Re}[E(\omega)]$ (red line) and the imaginary part $E_{2}=\operatorname{Im}$ (blue line) of the complex modulus $E(\omega)$ at 30 degrees Celsius. Points represent the measured values, the solid lines represent the fit obtained by using Eq. (3) .

needed to describe the test conditions, we exploit the Williams-Landel-Ferry relations [41] that, as described in Section 1, enable us to shift the data measured at different temperatures, thus obtaining the viscoelastic spectrum of the rubber, as shown in Figure 3 . Here, the experimental data, obtained in such a way, are fitted by means of the Eq. (3). The relaxation times are assumed to be in geometric progression with Euler's number as common ratio, i.e. $\tau_{k+1} / \tau_{k}=e$.

It is noteworthy that, as discussed in following paragraphs, the fit seems to effectively represent the measured data over the range of frequencies corresponding to the set of rolling speeds tested. As suggested in literature [48], measured and fitted data could show a certain discrepancy at high frequency because of slippage occurring in the sample grip of the DMA equipment. However, as shown below, this does not seem to be relevant for the rolling speed range under analysis.

\subsection{Temperature distribution}

By means of the experimental procedure described above, it is possible to obtain a detailed thermal map of the contact region. As shown in Figure 4, obtained for a normal load $P=2 \mathrm{~N}$ and a constant rolling speed $v=54$ $\mathrm{mm} / \mathrm{s}$, the temperature in the contact is higher than the room temperature $\left(T_{\text {room }}=30 \mathrm{C}\right)$. Furthermore, we can notice in the contact region the presence of hot spots, which are due to the surface roughness and correspond to asperities in the contact. This phenomenon of frictional heating is well known in sliding contacts (see [12] ) but is also present in rolling contacts.

It is interesting to consider how the temperature influences the contact solution and, specifically, when temperature heating can be neglected or, on the contrary, must be accounted for. The simplest way to approach this problem 


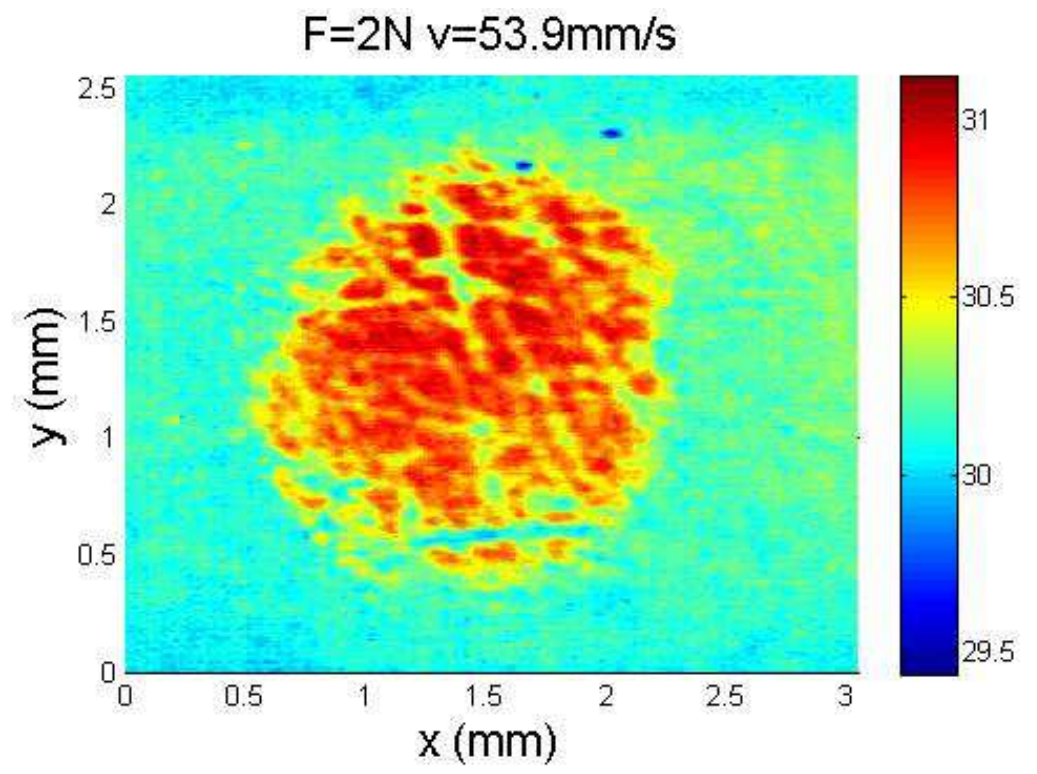

Figure 4: Temperature map for a constant normal load of $F_{N}=2 \mathrm{~N}$, a speed $v=54 \mathrm{~mm} / \mathrm{s}$ and a room temperature $T=30^{\circ} \mathrm{C}$.

is to focus on the mean temperature in the contact area since this may enable us to understand when thermal effects become prominent. For instance, Figure 5 , shows the mean temperature in the contact area as a function of the rolling speed for fixed a normal load $P=20 \mathrm{~N}$. Here, two regimes are clearly distinguished: for low speed values $(v<100 \mathrm{~mm} / \mathrm{s})$, temperature increase is negligible, but for higher speeds, there is a rapid growth in the temperature curve.

\subsection{Penetration}

Our experimental investigation also involves measuring the variation in penetration as a function of the rolling speed. This parameter has a strong importance in many applications since it is strictly related to the contact stiffness. Now, it is known that an increase in rolling speed causes a stiffening of the viscoelastic specimen [1]. Therefore, if the applied normal load is kept constant (e.g. equal to $F_{N}=20 \mathrm{~N}$ ), the penetration should show a decreasing trend with the speed. Indeed, this is what is reported in Figure 6, where the measured penetrations with varying speed is shown. Here, each experimental data point (red square in Figure 6) represents an average of 15 measurements, with a scatter of less than $5 \%^{1}$.

\footnotetext{
${ }^{1}$ Here, scatter is defined as $\sigma / \mu$, i.e. the ratio between the standard deviation $\sigma$ and the mean measured value $\mu$.
} 


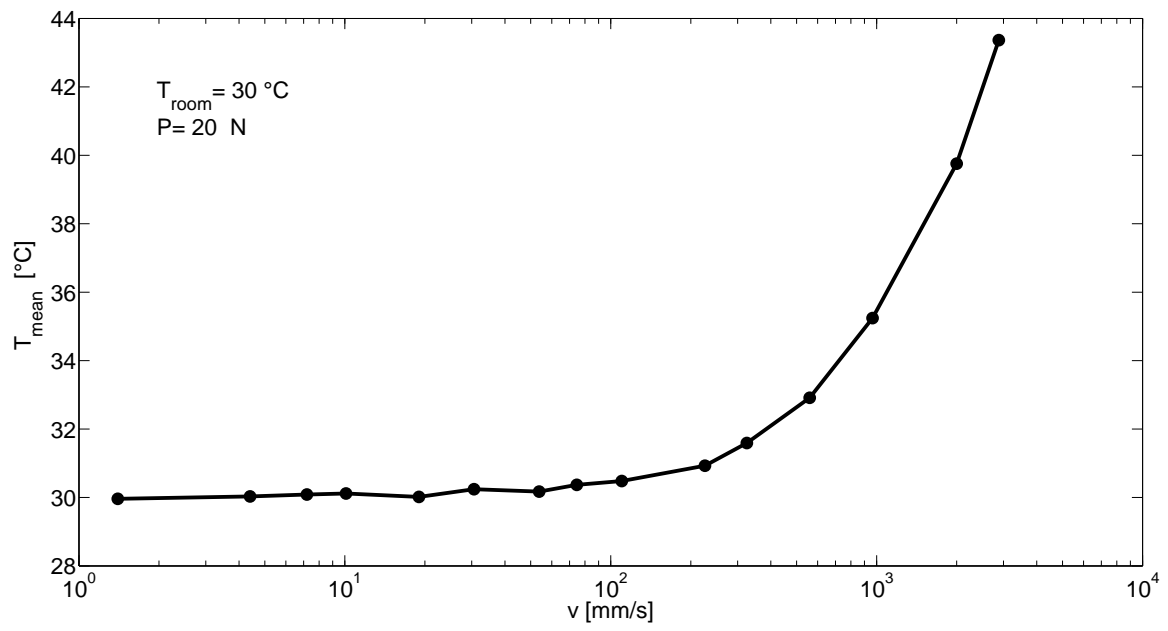

Figure 5: Mean temperature in the contact area as a function of the rolling speed $v$ for a constant normal load of $F_{N}=20 \mathrm{~N}$ and a room temperature $T=30{ }^{\circ} \mathrm{C}$.

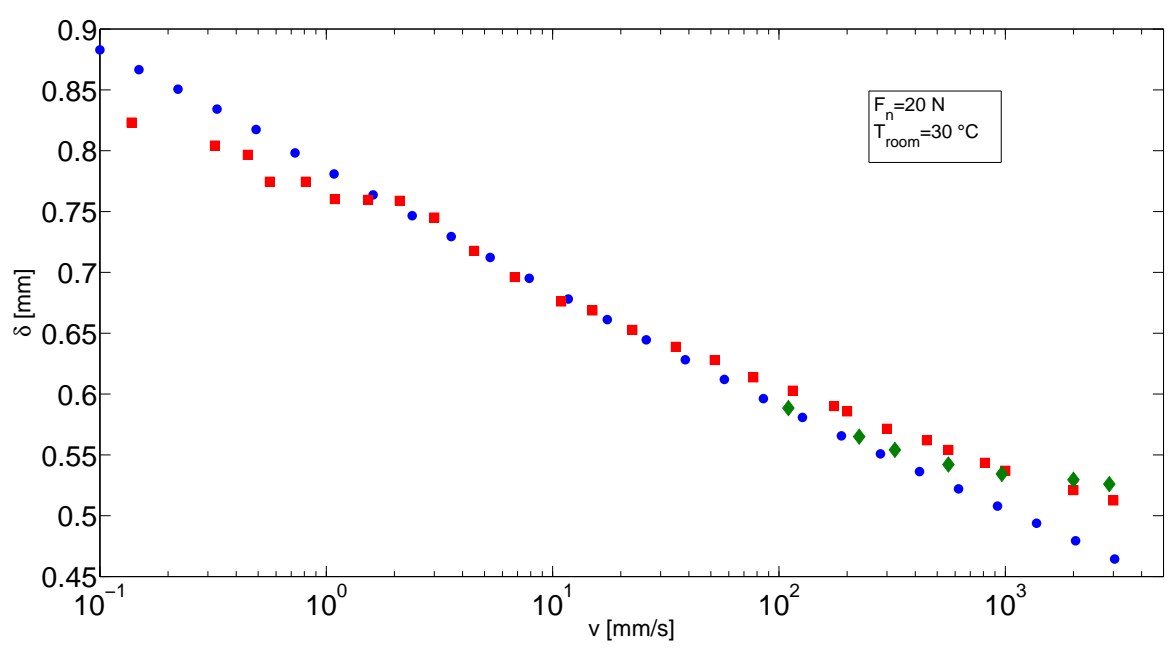

Figure 6: Contact penetration vs. rolling speed for a constant normal load of $F_{N}=20 N$ and a temperature $T=30^{\circ} \mathrm{C}$. Red squares refer to experimental outcomes, blue circle to numerical results obtained without any thermal corrections, greeen rhomboids to numerical simulations carried out with the viscoelastic response corrected with the temperature. 
If we compare the experimental results with the numerical predictions obtained using the viscoelastic response at the room temperature $T_{\text {room }}=30 \mathrm{C}$ (see blue circle in Figure 6), we find a good agreement over a very large speed range and, specifically, such a strong correlation is observed up to $100 \mathrm{~mm} / \mathrm{s}$. Actually, for very low speeds $(v<<1 \mathrm{~mm} / \mathrm{s})$, a certain discrepancy is found but, in our opinion, this could be due to the rig incapability of keeping constant so small speeds.

On the other side, for very high speeds $(v<<100 \mathrm{~mm} / \mathrm{s})$, experimental values tend to diverge from the numerical predictions. Indeed, we observe that, recalling thermal results shown in Figure 5, this divergence may be assumed to be caused by thermal heating effects occurring at high speed, which make the polymer softer and, hence, lead to higher than predicted penetration values. Consequently, a more accurate numerical approach should account for these temperature effects by considering thermal heating due to the material internal dissipation. The internal dissipation is related to the square of the strain rate in the material, thus leading to non-linear effect, which would strongly complicate the numerical approach. However, a rough procedure to take into account the thermal heating without fundamental changes to the numerical methodology proposed in [1] is to correct the viscoelastic modulus used in the numerical simulation, by inputting the value corresponding not to the room temperature, but to the mean contact temperature obtained experimentally. This approach can be applied to existing numerical results as shown as green diamonds in Figure 6 . Here, it can be seen that, at least in the speed range considered, the correction has the effect of extending the region of agreement between model and experiment to higher speeds. This somewhat validates the mean temperature approximation approach.

\subsection{Contact area}

The last part of our investigation deals with the study of the contact area. In Figure 7, we carry out a topographical analysis of this parameter: our aim is to evaluate the agreement between the borders extracted from the experimental snapshots, and the numerically evaluated shapes. Indeed, the two shapes match quite well, confirming the utility of the proposed BE method for a variety of problems, e.g. from seals to tyres technology [2] [3] . In all these cases, changes in the contact area obtained by varying the relative speed are of particular importance.

Even more important than the topographical results is the dimensional analysis of the contact area. In Figure 8, the contact area is shown as a function of the rolling speed. Here, we notice a clear decreasing trend, which is due to the material stiffening. In spite of the simplicity of the proposed setup, it is possible to study a large speed range covering more than four orders of magnitude. In the case of this set of measurements, the scatter is less than $8 \%$, which can be considered relatively low considering the convoluted nature of the acquisition and images processing procedures. Now, when focusing on the comparison with the numerical simulations, for rolling speeds up to $100 \mathrm{~mm} / \mathrm{s}$, there is a good agreement between experiments (blue squares) and numerical predictions (red 

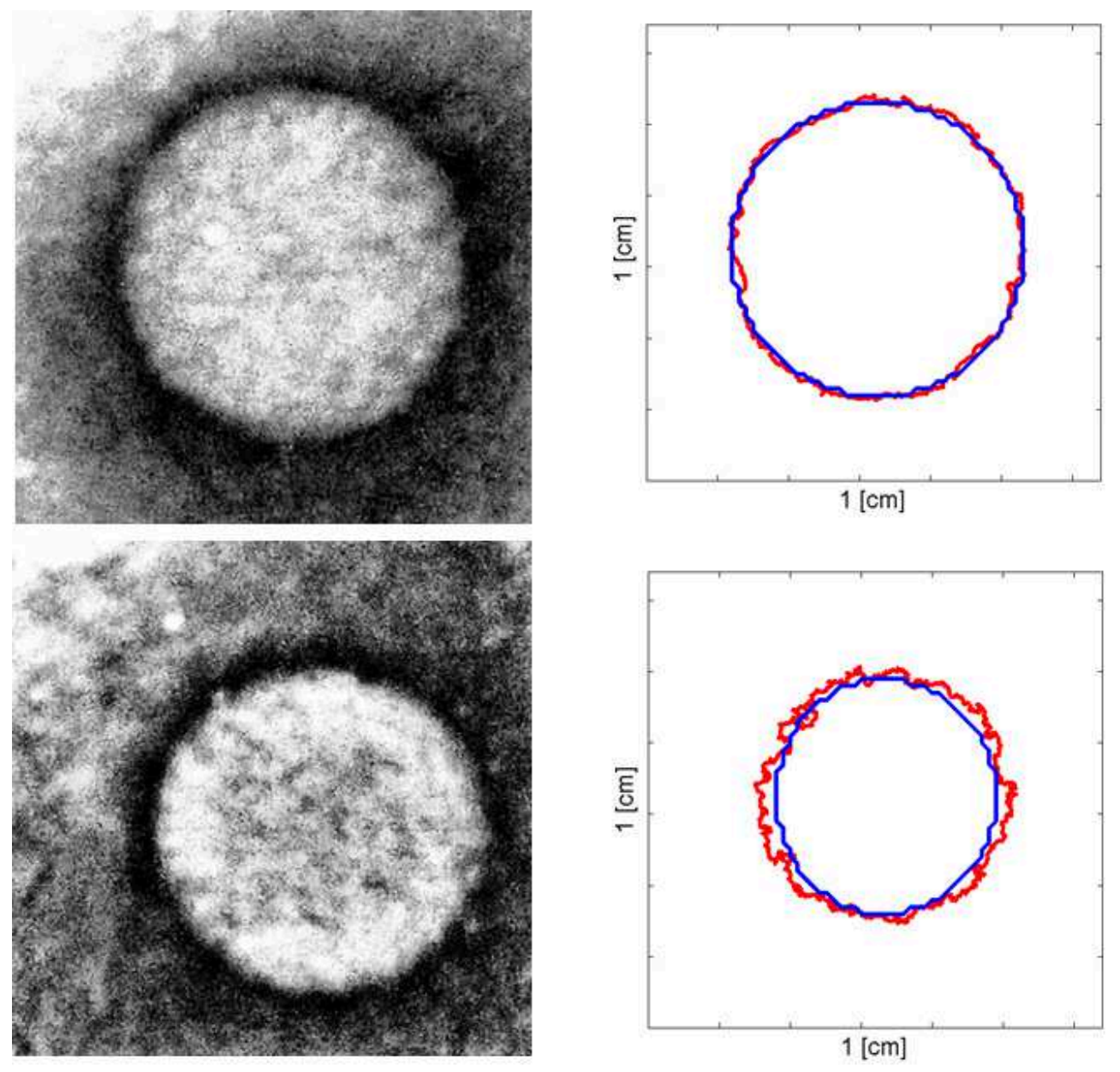

Figure 7: Contact area for speeds $0 \mathrm{~mm} / \mathrm{s}$ (first row) and $2 \mathrm{~mm} / \mathrm{s}$ (second row). Snapshots of the contact area are shown on the left while comparison between experimental (red line) and numerical (blue line) borders are shown on the right. 


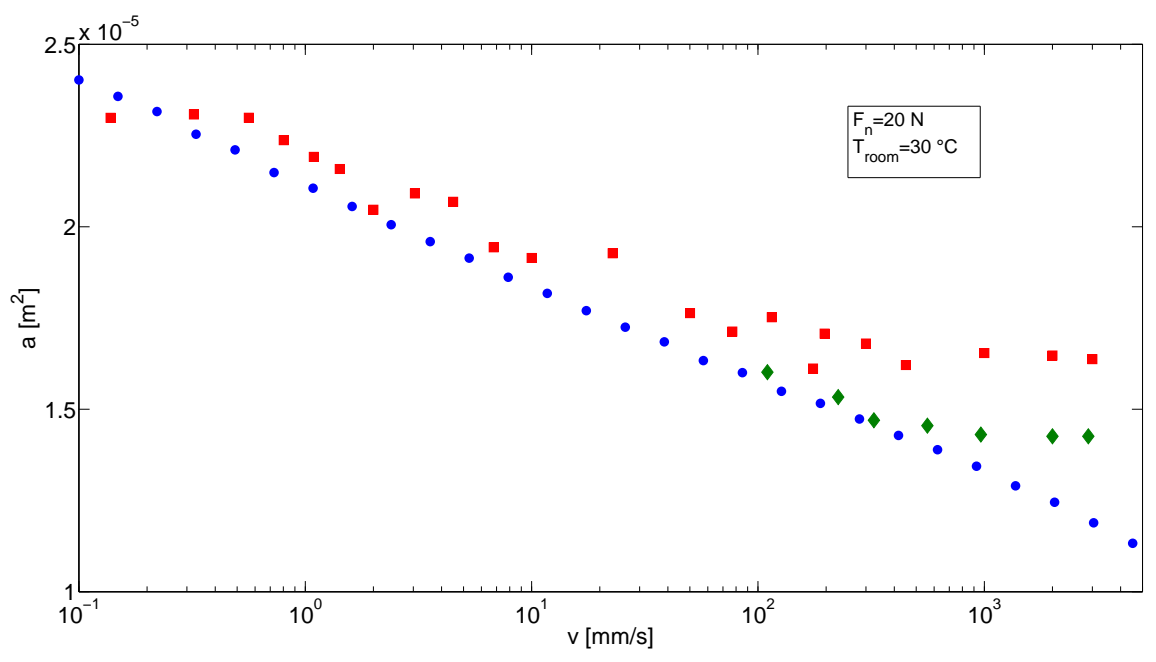

Figure 8: Contact area as a function of the rolling speed $v$ for a constant normal load of $F_{N}=20 \mathrm{~N}$ and a room temperature $T=30{ }^{\circ} \mathrm{C}$. Red squares refer to experimental outcomes, blue circle to numerical results obtained without any thermal corrections, greeen rhomboids to numerical simulations carried out with the viscoelastic response corrected with the temperature.

circles), whereas, at higher speeds, numerical outputs are lower than experimental values. By employing the same approach described above to account for thermal effects, we obtain the corrected values shown as green rhomboids in Figure 8. This method allow us to correctly predict the almost constant trend of the area with changing speed. However, values are still quantitatively lower than those experimentally measured. This may be due to the presence of hot spots contributions, which are neglected in the mean contact temperature approach. Furthermore, at these speeds, wear may have a certain importance also in rolling contacts and and may explain the larger values of contact areas than those predicted numerically.

\section{Conclusion}

In this paper, the main peculiarities marking viscoelastic rolling contact in terms of temperature, contact area and penetration have been experimentally evaluated. All these quantities have been compared with the numerical results obtained using the methodology presented by the authors in [1].

The temperature analysis has shown the presence of hot spots in the contact due to the presence of the surface roughness. Furthermore, when focusing on the mean temperature in the contact region, two different regimes have been found: given the test conditions ( room temperature $T_{\text {room }}=30 \mathrm{C}$ and normal load $P=20 \mathrm{~N}$ ), for rolling speeds up to $100 \mathrm{~mm} / \mathrm{s}$, the mean temperature is 
constant, whereas for higher speeds, a steep temperature increase is observed. Temperature effects, therefore, should be accounted for in this second region by carrying out a correlation between the contact solution and the material temperature. In this paper, we follow a straightforward, but approximate methodology that employs the mean temperature in the contact to correct the viscoelastic modulus used in the numerical simulations. Obviously, this approach neglects any thermal gradient and, therefore, any flash temperature effect, but noticeably it does not require significant modifications of the BE methodology.

Regarding the penetration and the contact area, in both cases, a descending trend due to the material stiffening is found. Furthermore, the direct comparison with numerical predictions is good up to $100 \mathrm{~mm} / \mathrm{s}$. Above this speed, both experimental penetration and contact areas are larger than the numerically predicted ones; such discrepancy can be attributed to the material heating and, to some extent, to wear phenomena. The mean temperature correction approach previously described to account for the heating has been useful to overcome, at least qualitatively, these problems. Indeed, the agreement between experiments and corrected numerical outcomes is very good for the penetration, while for contact area the approximate approach shows a qualitative improvement.

In conclusion, the validity of the BE method developed in[1] is confirmed for low speeds regime, where it provides reliable results . For higher speeds, temperature effects should be considered with a suitable thermo-mechanical analysis; however, the approximated approach that corrects the viscoelastic response according to the mean temperature may provide - at least qualitatevely - good results .

\section{Acknowledgements}

CP and GC thank the support of the Italian Ministry of Education, University and Research, within the Projects PON01 02238 and PON02 00576 3333604. CP gratefully acknowledges also the support of Marie Curie IEF fellowship SOFT-MECH (grant number 622632).

\section{References}

[1] G. Carbone, C. Putignano, A novel methodology to predict sliding/rolling friction in viscoelastic materials: theory and experiments. Journal of Mechanics and Physics of Solids,61 (8), pp. 1822-1834, (2013).

[2] Bottiglione F., Carbone G., Mangialardi L., Mantriota G., Leakage Mechanism in Flat Seals, Journal of Applied Physics 106 (10), 104902, (2009).

[3] O’Boy D.J., Dowling A.P., Tyre/road interaction noise-A 3D viscoelastic multilayer model of a tyre belt, Journal of Sound and Vibration, 322, 4-5, 829-850, (2009). 
[4] D. Craiem, R. L. Magin, Fractional order models of viscoelasticity as an alternative in the analysis of red blood cell (RBC) membrane mechanics, Phys. Biol. 7 013001, (2010).

[5] O. Regeva, S. Vandebrilb, E. Zussmana, C. Clasenb, The role of interfacial viscoelasticity in the stabilization of an electrospun jet, Polymer, 51, 12, 2611-2620, (2010).

[6] T. Tsujimotoa, H. Uyamaa, S. Kobayashib, Synthesis of high-performance green nanocomposites from renewable natural oils, Polymer Degradation and Stability, 95, 8, 1399-1405, (2010).

[7] Hunter S.C. , The rolling contact of a rigid cylinder with a viscoelastic half space Trans. ASME, Ser. E, J. Appl. Mech. 28, 611-617 (1961).

[8] M. Harrass, K.Friedrich , A.A.Almajid,Tribological behavior of selected engineering polymers under rolling contact, Tribology International,43, 635646, 2010.

[9] Grosch K. A. , The Relation between the Friction and Visco-Elastic Properties of Rubber, Proceedings of the Royal Society of London. Series A, Mathematical and Physical,274-1356, pp. 21-39, (1963).

[10] Persson B.N.J., Rolling friction for hard cylinder and sphere on viscoelastic solid, Eur. Phys. J. E 33, 327-333 (2010).

[11] Persson B. N. J., Theory of rubber friction and contact mechanics, J. Chem. Phys. 115, 3840 (2001).

[12] Persson B. N. J., Rubber friction: role of the flash temperature, J. Phys.: Condens. Matter 18 7789, (2006).

[13] Panek C. and Kalker J.J., Three-dimensional Contact of a Rigid Roller Traversing a Viscoelastic Half Space, J. Inst. Maths Applies 26, 299-313, (1980).

[14] J. Padovan, O. Paramadilok, Transient and steady state viscoelastic rolling contact, Comput Struct, 20, 545-553, 1984.

[15] Vollebregt E.A.H., User guide for CONTACT, J.J. Kalker's variational contact model, Technical Report TR09-03, version 1.18

[16] Goriacheva, G.: Contact problem of rolling of a viscoelastic cylinder on a base of the same material. J. Appl. Math. Mech. 37(5), 925933 (1973). doi:10.1016/0021-8928(73)90017-8

[17] E. H. Lee and J. R. M. Radok The Contact Problem for Viscoelastic Bodies. J. Appl. Mech., 27, 3, 438-444,1960.

[18] Christensen R. M., Theory of viscoelasticity,Academic Press, New York 
[19] J. Padovan. Finite element analysis of steady and transiently moving/rolling nonlinear viscoelastic structure-I. theory. Computers \& Structures, 27(2):249-257, 1987.

[20] J. Padovan, A. Kazempour, F. Tabaddor, and B. Brockman. Alternative formulations of rolling contact problems. Finite Elements in Analysis and Design, 11:275-284, 1992.

[21] L. Nasdala, M. Kaliske, A. Becker, H. Rothert, An efficient viscoelastic formulation for steady-state rolling structures, Computational Mechanics 22, 395-403, 1998.

[22] Le Tallec P., Rahler C., Numerical models of steady rolling for non-linear viscoelastic structures in finite deformations, International Journal for $\mathrm{Nu}-$ merical Methods in Engineering, vol. 37, 1159-1186 (1994).

[23] J. Halaunbrenner, A. Kubisz: ASLE-ASME Lubrication Conf., Chicago (1967) Paper No. 67-Lub-25.

[24] Johnson K.L.J., Contact Mechanics, Cambridge University Press (1985).

[25] B. A. Krick, Jennifer R. Vail, Bo N. J. Persson, W. G. Sawyer, Optical In Situ Micro Tribometer for Analysis of Real Contact Area for Contact Mechanics, Adhesion, and Sliding Experiments. Tribol Lett. DOI 10.1007/s11249-011-9870-y

[26] B. Lorenz, B.N.J. Persson, S. Dieluweit, and T. Tada, Rubber friction: Comparison of theory with experiment, DOI 10.1140/epje/i2011-11129-1 Eur. Phys. J. E (2011) 34: 129 THE EUROPEAN PHYSICAL JOURNAL E.

[27] D. Maugis, Adhesion of Spheres: The JKR-DMT Transition Using a Dugdale Model, Journal of Colloid and Interface Science, 150, 1, (1992).

[28] G. L. Wannop, J. F. Archard. Elastic Hysteresis And A Catastrophic Wear Mechanism For Polymers. Proc. I Mech E 187 p615- (1973).

[29] John D. Ferry, Viscoelastic Properties of Polymers, John Wiley \& Sons, Inc.

[30] Putignano C, Reddyhoff T, Carbone G, Dini D (2013). Experimental Investigation of Viscoelastic Rolling Contacts: A Comparison with Theory. Tribology Letters, vol. 51, p. 105-113, ISSN: 1023-8883, doi: 10.1007/s11249013-0151-9.

[31] Nackenhorst U, The ALE-formulation of bodies in rolling contact Theoretical foundations andfinite element approach, Comput Method Appl M, 193, 4299-4322, 2004. 
[32] D. Felhõs, D. Xu1, A. K. Schlarb, K. Váradi, T. Goda, Viscoelastic characterization of an EPDM rubber and finite element simulation of its dry rolling friction, eXPRESS Polymer Letters 2, 3,157-164, 2008 .

[33] Dumitru N. Olaru, Ciprian Stamate, Gheorghe Prisacaru, Rolling Friction in a Micro Tribosystem, Tribol Lett , 35:205-210, 2009 ..

[34] S. Yoneyama, J. Gotoh and M. Takashi, Experimental Analysis of Rolling Contact Stresses in a Viscoelastic Strip, Volume 40, Number 2, 203-210, DOI: $10.1007 / \mathrm{BF} 02325047$.

[35] Putignano C., Afferrante L., Carbone G., Demelio G. A new efficient numerical method for contact mechanics of rough surfaces. International Journal of Solids and Structures, 49 (2), 338-343, DOI 10.1016/j.ijsolstr.2011.10.009, (2012).

[36] Putignano C. , Afferrante L. , Carbone G. , . Demelio G, The influence of the statistical properties of self-affine surfaces in elastic contact: a numerical investigation, in press, Journal of Mechanics and Physics of Solids, (2012).

[37] Putignano C, Afferrante L, Carbone G, Demelio GP (2013). A multiscale analysis of elastic contacts and percolation threshold for numerically generated and real rough surfaces. Tribology International, vol. 64, p. 148-154, ISSN: 0301-679X, doi: 10.1016/j.triboint.2013.03.010.

[38] Reddyhoff T, Spikes HA, Olver AV. (2009). Improved Temperature Mapping of EHL Contacts. Proc. I.Mech.E. part J: Journal of Engineering Tribology, vol. 223, p. 1165-1177, doi: 10.1243/13506501JET499.

[39] Ingram M, Reddyhoff T, Spikes HA, (2010). Thermal Behaviour of a Slipping Wet Clutch Contact. Tribology Letters, vol. 42, 1, p. 23-32, doi: 10.1007/s11249-010-9669-2.

[40] Le Rouzic J, Reddyhoff T, (2013). Development of Infrared Microscopy for Measuring Asperity Contact Temperatures. ASME Journal of Tribology, vol. 135, 021504, doi: 10.1115/1.4023148.

[41] M.L. Williams, R.F. Landel, J.D. Ferry, J. Amer. Chem. Soc., 77:3701, 1955.

[42] Carbone G., Lorenz B., Persson B.N.J. and Wohlers A., Contact mechanics and rubber friction for randomly rough surfaces with anisotropic statistical properties, The European Physiscal Journal E - Soft Matter, 29 (3), 275284, (2009)

[43] Carbone G., Mangialardi L., Adhesion and friction of an elastic half-space in contact with a slightly wavy rigid surface, Journal of the Mechanics and Physics of Solids, 52 (6), 1267-1287, 2004. 
[44] Persson, B.N.J., Rubber friction: Role of the flash temperature, Journal of Physics Condensed Matter, 18 (32), 7789-7823, (2006)

[45] Lorenz B.; Persson B. N. J.; Dieluweit S., tada T., Rubber friction: Comparison of theory with experiment, EUROPEAN PHYSICAL JOURNAL E 34 (12), 129, doi: 10.1140/epje/i2011-11129-1 (2011).

[46] Carbone G., Mangialardi L.: Analysis of adhesive contact of confined layers by using a Green's function approach, The Journal of the Mechanics and Physics of Solids, 56 (2), 684-706 (2008).

[47] J de Vicente, J R Stokes, and H A Spikes, Rolling and sliding friction in compliant, lubricated contact, Proc. IMechE Vol. 220 Part J: J. Engineering Tribology.

[48] Michael Kessler, Advanced Topics in Characterization of Composites, Trafford Publishing, 2004.

[49] Carbone G., Scaraggi M., Tartaglino U. "Adhesive contact of rough surfaces: comparison between numerical calculations and analytical theories", The European Physiscal Journal E - Soft Matter, 30 (1), 65-74 (2009) 